\title{
The experience of infection prevention for coronavirus disease 2019 (COVID-19) during general anesthesia in an epidemic of COVID-19: including unexpected exposure case - Two cases report -
}

Received May 21, 2020

Revised July 7, 2020

Accepted July 13, 2020

\section{Hyunkyum Kim, Jeongmin Oh, Sang-Gon Lee, and Kwang-Seok Shim}

Department of Anesthesiology and Pain Medicine, Daegu Fatima Hospital, Daegu, Korea

\author{
Corresponding author \\ Kwang-Seok Shim, M.D. \\ Department of Anesthesiology and \\ Pain Medicine, Daegu Fatima \\ Hospital, 99, Ayang-ro, Dong-gu, \\ Daegu 41199, Korea \\ Tel: 82-53-940-7434 \\ Fax: 82-53-940-7443 \\ E-mail: allforone97@naver.com
}

Background: Coronavirus disease 2019 (COVID-19) has been an epidemic in the Republic of Korea since the first patient who came in China was confirmed as having the disease on January 19, 2020. Therefore, surgery for COVID-19-related patients is expected to increase.

Case: We experienced two cases of COVID-19-related patients who underwent surgery for femur fracture under general anesthesia. In one case, infection control to prevent transmission was achieved, while in the second case, healthcare workers were exposed to severe acute respiratory syndrome coronavirus-2 (SARS-CoV-2).

Conclusions: In areas where there is COVID-19 epidemic, SARS-CoV-2 real-time polymerase chain reaction should be performed before surgery in patients with symptoms of respiratory infection such as fever, cough, and sputum, or for those in whom the possibility of COVID-19 cannot be completely excluded. Efforts should be made to prevent healthcare worker's exposure and the contamination of the operating room.

Keywords: COVID-19; Epidemics; General anesthesia; Infection control; Personal protective equipment; SARS-CoV-2.
Coronavirus disease 2019 (COVID-19) is a disease caused by the severe acute respiratory syndrome coronavirus 2 (SARS-Cov-2) infection. In the Republic of Korea, 10,936 patients had been diagnosed and 258 patients had died of COVID-19 as of May 12 of 2020, since the first case was diagnosed on January 19, 2020 [1].

If a COVID-19-related patient undergoes surgery in areas where COVID-19 is prevalent, healthcare workers should perform infection control during aerosol-generating procedures to minimize the transmission by airborne viral spread and contact transmission by respiratory droplets. Therefore, when performing surgery for COVID-19-related patients at our hospital, healthcare workers wore an appropriate personal protective equipment (PPE) to prevent exposure to SARS-Cov-2. Anesthesia was also performed in a negative pressure operating room designed to prevent infection with minimal healthcare workers.

We performed general anesthesia for femoral fracture surgery in two COVID-19-related patients. Herein, we report a case in which infection control was performed and another case in which healthcare workers were exposed to SARS-CoV-2.

The study was approved by the Institutional Review Board of the Daegu Fatima Hospita (no. DFE20ORIO064).

This is an Open Access article distributed under the terms of the Creative Commons Attribution Non-Commercial License (http://creativecommons.org/licenses/by-nc/4.0) which permits unrestricted non-commercial use, distribution, and reproduction in any medium, provided the original work is properly cited.

Copyright (C) the Korean Society of Anesthesiologists, 2020 
We obtained informed consent from the patients after surgery to publish this report.

\section{CASE REPORT}

\section{Case 1}

An 88-year-old male patient without any underlying disease, who had been taking drugs due to symptoms of cough, sputum and myalgia a week prior to the visit to the hospital, came to our emergency room because fell from his height. At the time of the visit, his mental status was drowsy, and a fever of $37.7^{\circ} \mathrm{C}$ was observed. Based on brain magnetic resonance imaging, an acute cerebral infarction was suspected. A left femur intertrochanteric fracture was observed on the radiography of the lower limbs. There were no specific findings on the chest X-ray and blood tests, but SARS-CoV-2 quantitative real-time polymerase chain reaction (RT-PCR) and chest computed tomography (CT) were performed due to symptoms of respiratory infection such as cough, sputum and fever. Chest CT showed consolidation in both lower lungs which was suspected to be pneumonia (Fig. 1), and a positive result of the SARSCoV-2 RT-PCR was confirmed. The patient was admitted to an isolation ward and treated for acute cerebral infarction.

The patient was confirmed to be infected with SARS$\mathrm{CoV}-2$, but a bacterial pneumonia was not excluded; therefore, antibiotics and hydroxychloroquine were adminis-

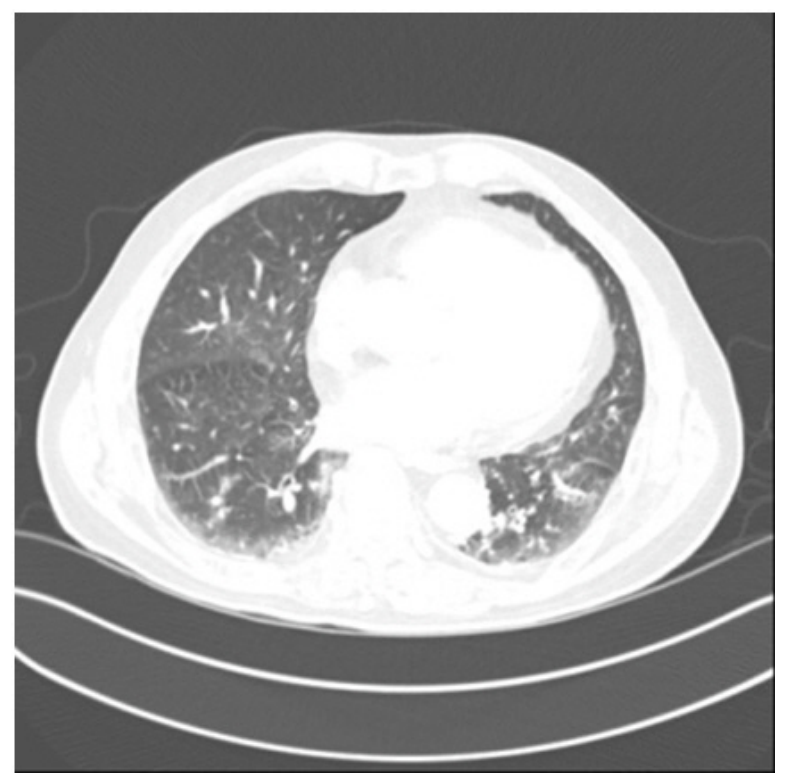

Fig. 1. Chest CT showed consolidations in both lower lung which were suspected as pneumonia. CT: computed tomography. tered. Two RT-PCR tests performed on the 6th and 10th days of admission showed negative results. After 2 weeks of admission, there was no evidence of active infection on chest CT; therefore, it was decided to perform a closed reduction with internal fixation of the left femur intertrochanteric fracture on the 19th days of admission. Since the patient was taking an antiplatelet agent (clopidogrel) due to the cerebral infarction, we decided to administer a general anesthesia to the patient. According to our hospital's COVID-19 response guidelines (Fig. 2) based on previous results of SARS-CoV-2 positive patient reports, we decided to wear enhanced PPEs.

A separate negative pressure zone was set to prevent aerosol spread. We covered the anesthesia machine, an operating bed, and equipment in the operating room with vinyl. To prevent contamination of the anesthesia machine, high-efficiency particulate air (HEPA) filters (GVS Filter Technology UK Ltd., UK) were applied to the inspiratory and expiratory limbs of the breathing circuit and the patient's side was connected to the endotracheal tube. Healthcare workers wore enhanced PPEs including N95 respirator, surgical cap, double gown, double glove, shoe covers, and powered air-purifying respirators (PAPR), which was separately set in the negative pressure zone. The patient was moved to the operating room with healthcare workers wearing the enhanced PPE. After entering the operating room, we continuously monitored the electrocardiogram (EKG), pulse oximetry, and blood pressure. After preoxygenation, propofol $1.5 \mathrm{mg} / \mathrm{kg}$ was administered for anesthesia induction. Rocuronium bromide $0.8 \mathrm{mg} / \mathrm{kg}$ was administered to induce a neuromuscular blockade sufficient to prevent the spread of airborne droplets. Tracheal intubation was performed using a McGrath MAC (McGRATH, Aircraft Medical Ltd., UK) videolaryngoscopy. After intubation, we closed the operating room for $30 \mathrm{~min}$ to prevent aerosol transmission. Anesthesia was maintained with 1.5-2.0 vol\% sevoflurane and remifentanil infusions.

After $45 \mathrm{~min}$, the surgery was completed and sugammadex $2 \mathrm{mg} / \mathrm{kg}$ was administered to reverse the neuromuscular blockade. Five minutes after Sugammadex administration, the neuromuscular blockade was sufficiently reversed such that tracheal tube extubation was performed. After extubation, the operating room was closed for another 30 min to prevent aerosol transmission. Then, to prevent the post-anesthesia care unit (PACU) from being contaminated, the patient had to recover in the operating room. After recovering for $30 \mathrm{~min}$, healthcare workers wearing en- 

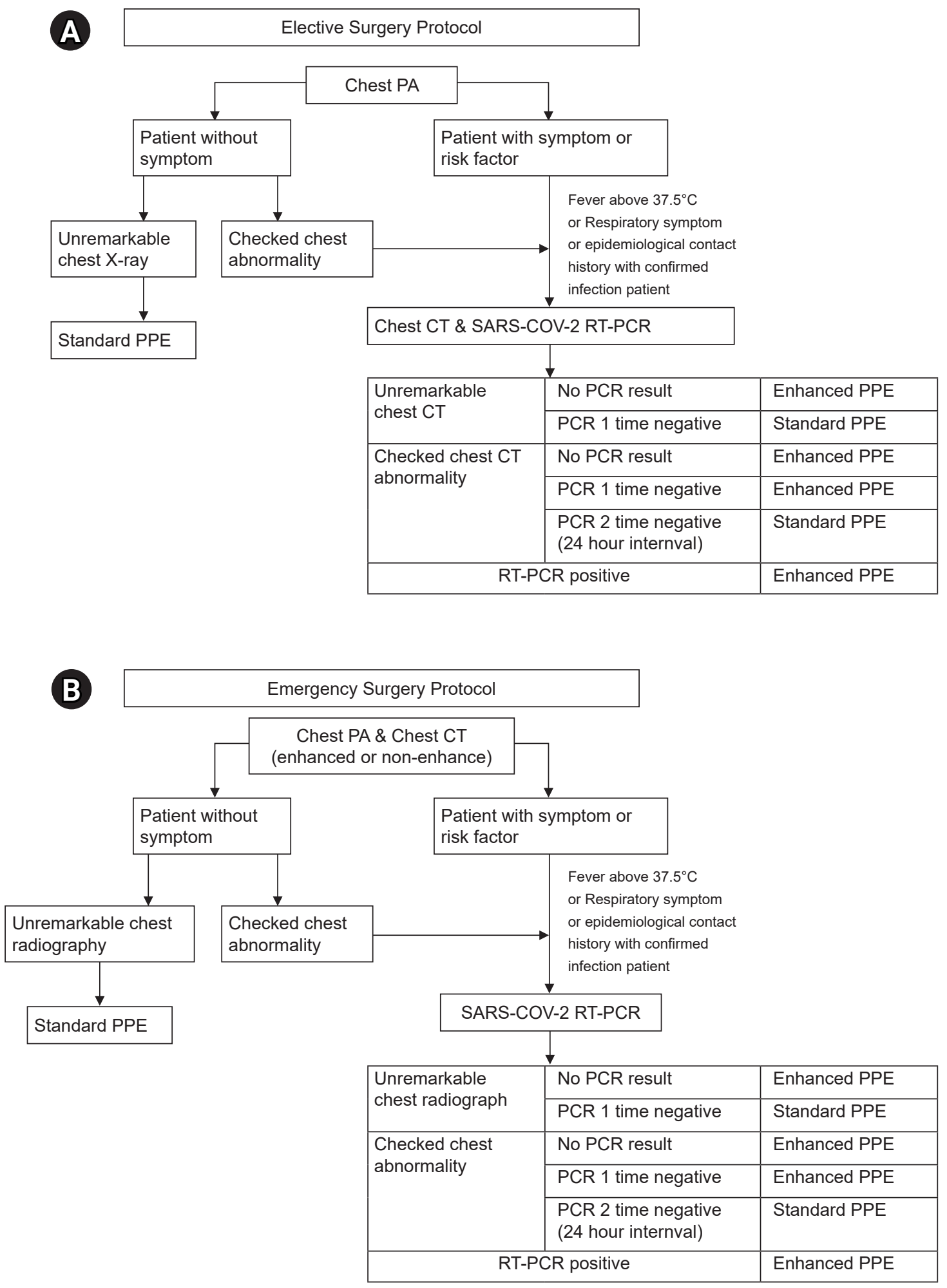

Fig. 2. (A) When planning elective surgery, Chest X-ray was performed first. If the possibility of SARS-CoV-2 infection could not be completely ruled out, chest CT and RT-PCR were also performed. (B) In the case of emergency surgery, chest CT was checked as well as chest X-ray in all patients. SARS-CoV-2 PCR was performed when abnormality in Chest CT was detected, patients had risk factors, or respiratory symptoms such as cough and sputum. RT-PCR: real-time polymerase chain reaction, PPE: personal protective equipment, SARS-CoV-2: severe acute respiratory syndrome coronavirus-2, COVID-19: coronavirus disease 2019, CT: computed tomography, PA: posteroanterior. 
hanced PPEs moved the patient back to the isolation ward through a separate elevator. After the surgery was completed, healthcare workers who participated in the surgery removed the enhanced PPE in the anteroom of the negative pressure zone, and the operating room's surface was disinfected and the operating room was ventilated for $30 \mathrm{~min}$.

A week after the surgery, the chest CT result of the patient did not show any specific findings and the SARSCoV-2 RT-PCR was also confirmed to be negative. Therefore, eight days after the surgery, the patient was discharged with no other complications.

\section{Case 2}

A 73-year-old female patient was admitted for further evaluation due to a suspected right lesser trochanter pathologic fracture on the hip X-ray and bone metastasis on the whole body bone scan. The patient was on medication for hypertension and hypothyroidism. She was also diagnosed with pulmonary tuberculosis 10 years ago and was cured after treatment. The patient had been taking antiplatelet agents for 9 years after intra-arterial stent insertion due to right internal carotid artery stenosis. SARSCoV-2 RT-PCR was performed because the patient had a fever of $37.8^{\circ} \mathrm{C}$ one week before admission. A negative result was reported. On the second day of admission, a fever of $38^{\circ} \mathrm{C}$ or higher was observed; thus, SARS-CoV-2 RT-PCR was performed but negative results were confirmed. Chest radiography showed no specific findings other than the old pulmonary tuberculosis lesion in the right upper lung. Later, the fever subsided.
On the 4th day of admission, chest CT showed no specific findings except for the multifocal post-infectious old lesions in the right lung, and there was no change compared to the previous chest CT (Fig. 3A). Therefore, on the 5th day of admission, an excisional biopsy of the right femur was performed. Considering that the possibility of COVID-19 infection was excluded, the patient was transferred to a room different from the previous one after the excisional biopsy was performed. After the biopsy, a fever of $37.5^{\circ} \mathrm{C}$ or higher was noted and the C-reactive protein was elevated. As a result of the biopsy, a lymphoma was suspected.

On the 9th day of admission, a right femoral shaft pathologic fracture occurred, and it was decided to perform a closed reduction of the fracture with internal fixation at the emergency on that same day. We considered that the patient had been confirmed to be negative for SARS-CoV-2 by RT-PCR 6 days before admission and on the 2nd day of admission, and there was no evidence of active infection on chest CT on the 4th day of admission. For the persistent fever, we consulted with the division of infectious disease and infection control department. The division of infectious disease and infection control department suggested that the patient's fever seemed to be a neoplastic fever caused by lymphoma. Therefore, we excluded the possibility of a SARS-CoV-2 infection in the patient and decided to perform the surgery with standard PPEs including surgical gloves, gown, eye shields, and N95 respirators. Since the patient was taking an antiplatelet agent (clopidogrel) due to internal carotid artery stenosis, we decided to administer a general anesthesia to the patient. After preoxygenation, propofol $2 \mathrm{mg} / \mathrm{kg}$ was administered for anesthesia
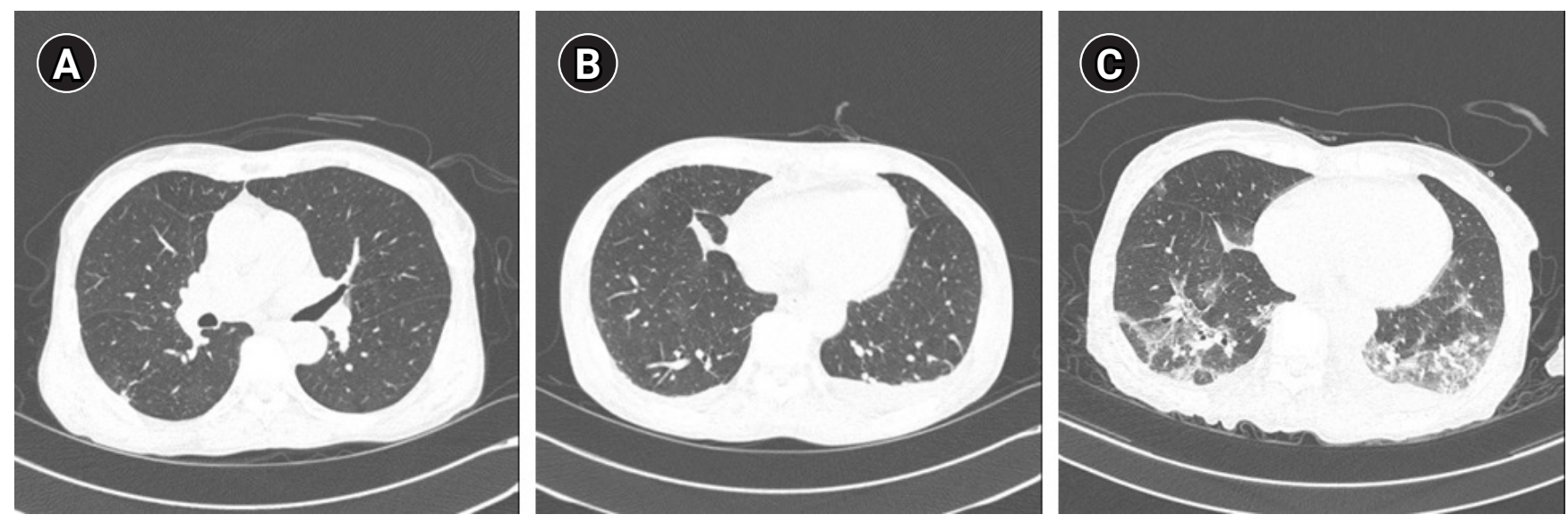

Fig. 3. (A) Chest CT showed no evidence of active infection and no change in the interval of old tuberculosis. (B) Chest CT showed increased bilateral pleural effusion and no interval change of GGA in the right lower lung. (C) Chest CT showed a multifocal patchy GGA lesion with interlobular septal thickening that appears to be a new suspected viral pneumonia. CT: computed tomography, GGA: ground-glass appearance. 
induction, and rocuronium bromide $0.6 \mathrm{mg} / \mathrm{kg}$ was administered. Tracheal intubation was performed using McGrath MAC (McGRATH, Aircraft Medical Ltd.) and anesthesia was maintained with 1.5-2.0 vol\% sevoflurane and remifentanil infusion. Oxygen saturation was maintained at $100 \%$ during anesthesia, and blood pressure, heart rate, and mechanical ventilation were maintained stable.

The surgery lasted $85 \mathrm{~min}$. Then, sugammadex $2 \mathrm{mg} / \mathrm{kg}$ was administered to reverse the neuromuscular blockade. Five minutes after administration of sugammadex, the neuromuscular blockade was completlely confirmed; therefore, tracheal tube extubation was performed. After that, the patient was transferred to the PACU for recovery. After an hour, the PAR score was checked at 9 points; therefore, the patient was moved to the hospital room.

One day after the surgery, the patient developed a fever of $38.4^{\circ} \mathrm{C}$ again, and another patient in the same hospital room was confirmed to be COVID-19 positive. Therefore, the chest CT and SARS-CoV-2 RT-PCR test were redone on the patient. On chest CT, bilateral pleural effusion was observed (Fig. 3B), and the SARS-CoV-2 RT-PCR results were positive. As a result, the patient was confirmed to be COVID-19 positive and moved to the isolated room. After that, Lopinavir, ritonavir, and hydroxychloroquine were administered for COVID-19 treatment and antibiotics were administered in consideration of possible bacterial pneumonia.

We investigated healthcare workers who participated in the surgery. In the PACU, all healthcare workers were wearing standard PPEs, so there were no exposed healthcare workers, but confirmed that the anesthesiologist and nurse who participated in the surgery were exposed because they did not wear eye shields when they performed the anesthesia. Therefore, we ran SARS-CoV-2 RT-PCR on these two healthcare workers, and made them stay at home for two weeks. For the two tests, both showed negative results on SARS-CoV-2 RT-PCR. Fever and cough symptoms were not observed for two weeks. After two weeks, on the SARSCoV-2 RT-PCR, both healthcare workers' results were reported as negative.

By the way, the operating room was also exposed to the possibility of infection. Therefore, we performed surface disinfection, emptied the operating room for $24 \mathrm{~h}$, and performed room air ventilation. A carbon dioxide absorber of the anesthesia machine was also replaced as we performed disinfection. 5 days after the surgery, a multifocal patchy ground-glass appearance (GGA) lesion in both lungs which were suspected to be viral pneumonia was reported on the chest CT scan (Fig. 3C); therefore, antibiotics and hydroxychloroquine were administered. However, pneumonia had not improved, and the patient was given a ventilator care in the intensive care unit. Twenty-three days after surgery, pneumonia was aggravated and the patient was died.

\section{DISCUSSION}

COVID-19 is a disease caused by a new virus, SARSCoV-2, which occurred in December 2019 in Wuhan, Hubei Province, China [2]. On March 11, 2020, the World Health Organization (WHO) declared a global pandemic of SARSCoV-2 spreading across the world. Like SARS-CoV, SARS$\mathrm{CoV}-2$ can cause person-to-person transmission by airborne viral spread, physical contact, and respiratory droplets $[3,4]$.

In aerosol-generating procedures such as tracheal intubation, noninvasive ventilation and cardiopulmonary resuscitation, the risk of coronavirus transmission can be increased $[5,6]$. In particular, when performing general anesthesia in the operating room, aerosol occurs in a limited space so there is a higher risk of healthcare workers being exposed to infection. Due to the increased risk of SARSCoV-2 transmission during general anesthesia, in order to prevent transmission of SARS-CoV-2 during the surgery of COVID-19-related patients, we planned to prepare a negative pressure operating room, healthcare workers wearing appropriate PPE, and minimize in-hospital exposure.

During the aerosol-generating procedure in a negative pressure room, WHO recommends both performing air changes at least 12 times per hour and controlling the direction of air flow during mechanical ventilation at the same time [6]. Our hospital performs air changes at least 12 to 15 times per hour in the operating room, but it does not have any permanent negative pressure operating rooms. Thus, we converted two operating rooms into temporary negative pressure operating rooms for COVID-19 related patients by referring to the setting of a negative pressure operating room during the Middle East respiratory syndrome epidemic [7]. To do this, the operating room was divided into two rosettes and the hospital clean unit setting was changed. The fresh air inflow of operating rooms was maximized in rosette A, and minimized (50\% of maximum) in rosette B (Fig. 4) The outflow of both rosettes remained constant.

With this setting, Rosette A was set as a positive pressure 


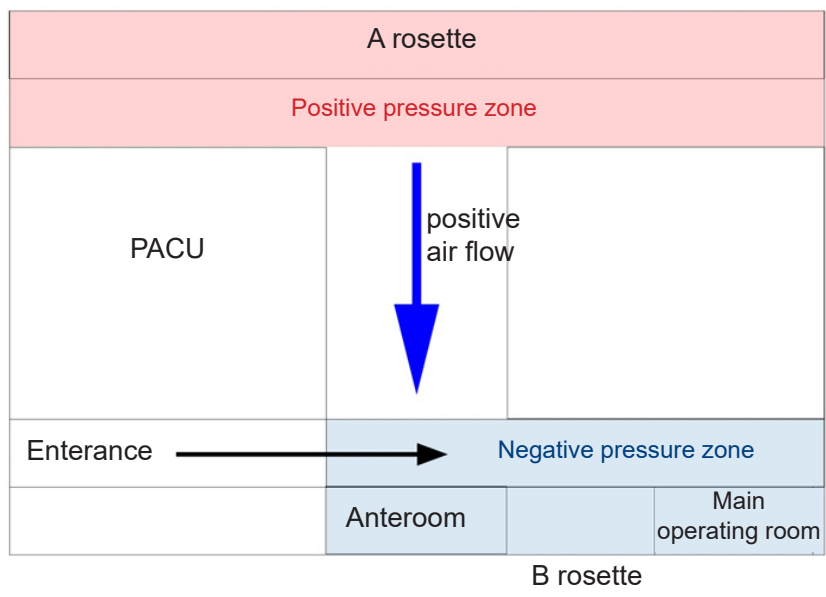

Fig. 4. This picture is a schematic diagram of our operating hall. We set up rosette $A$ and rosette $B$. The rosette close to the entrance was set as a negative pressure zone. PACU: post-anesthesia care unit.

zone, and rosette B was set as a relatively negative pressure zone. This caused the air to flow only in one direction between the two zones. Whenever a patient is about to move to the operating room, a smoke test was performed to ensure that negative pressure was established (Supplementary Video 1). According to the operating room response guidelines, we consulted with the division of infectious disease and infection control department and allowed healthcare workers to wear standard PPE or enhanced PPE when participating in surgery (Fig. 2).

We checked whether there was an abnormality on chest radiography, which is basically performed as a preoperative evaluation. This is because, at the time, many SARSCoV-2 infections occurred epidemically in Daegu, Republic of Korea, making it difficult to perform SARS-CoV-2 RTPCR in all patients undergoing surgery. Although chest X-ray was less sensitive in detecting COVID-19 than chest $\mathrm{CT}$, since it was performed by preoperative evaluation in all patients, the possibility of COVID-19 could be evaluated first through chest X-ray [8].

Xie et al. [9] reported that chest CT can also help detect COVID-19 in patient $s$ with negative results in SARS-CoV-2 RT-PCR. Therefore, we also performed chest CT in patients with risk factors, or with symptoms of respiratory infection such as fever, cough, and sputum.

SARS-CoV-2 RT-PCR is considered a standard for the detection of SARS-CoV-2 infection, but false-negative results in sampling should also be considered. When collecting a sample of RT-PCR, errors may occur depending on the examiner, and a sufficient amount of sample may not be collected from the upper respiratory tract, resulting in weakly positive results. In this case, RT-PCR should be performed by sampling from the lower respiratory tract, but since it was not easy to perform this, chest CT was performed to check the possibility of SARS-CoV-2 infection more closely [10].

When performing urgent surgery without performing SARS-CoV-2 RT-PCR or chest CT, the possibility of COVID-19 could not be completely excluded. Therefore, all healthcare workers wore enhanced PPE. The surgery was performed in a negative pressure zone. When performing surgery other than urgent surgery, we immediately received a radiologist's report on chest radiography or chest CT scans of patients who underwent surgery because of the consultation with the radiology department in advance. Since it was difficult to completely exclude the possibility of COVID-19 as a result of one negative RT-PCR [10], all healthcare workers wore standard PPE to performed anesthesia even when not wearing enhanced PPE.

The standard PPE includes surgical gloves, gown, eye shields, and N95 respirators (Fig. 5) while enhanced PPE includes overall clothes with head cover, shoe covers, goggles, two pairs of surgical gloves, a PAPR (Fig. 6). Healthcare workers wore enhanced PPEs in separate anterooms. Even after the surgery, healthcare workers discarded the PPEs in the anteroom, performed basic disinfection, and then left. To minimize virus transmission in the hospital, surgery was planned after completion of the elective surgery. The patient was moved to the operating room through separate elevators and pathways. The pathway around the negative pressure operating room was also controlled. After the aerosol-generating procedure of endotracheal intubation and extubation, the operating room was closed and room ventilation was performed for $30 \mathrm{~min}$. It has been reported that the stability of SARS-CoV-2 is maintained for $3 \mathrm{~h}$ in aerosol and $72 \mathrm{~h}$ in materials such as plastic or stainless steel [11]. Therefore, the anesthesia machine and operating bed in the operating room are covered with vinyl for protection. After the surgery, the operating room was ventilated for more than $3 \mathrm{~h}$, and had not been used for at least another $24 \mathrm{~h}$. The operating room and anesthetic machine were disinfected and extra surface disinfection was performed.

Anesthesia was performed by a well-trained anesthesiologist, and intubation was performed using videolaryngoscopy to minimize the possibility of intubation failure.

In case 1 , it was a surgery of COVID-19 confirmed patient; therefore, the protocol was well performed and anes- 


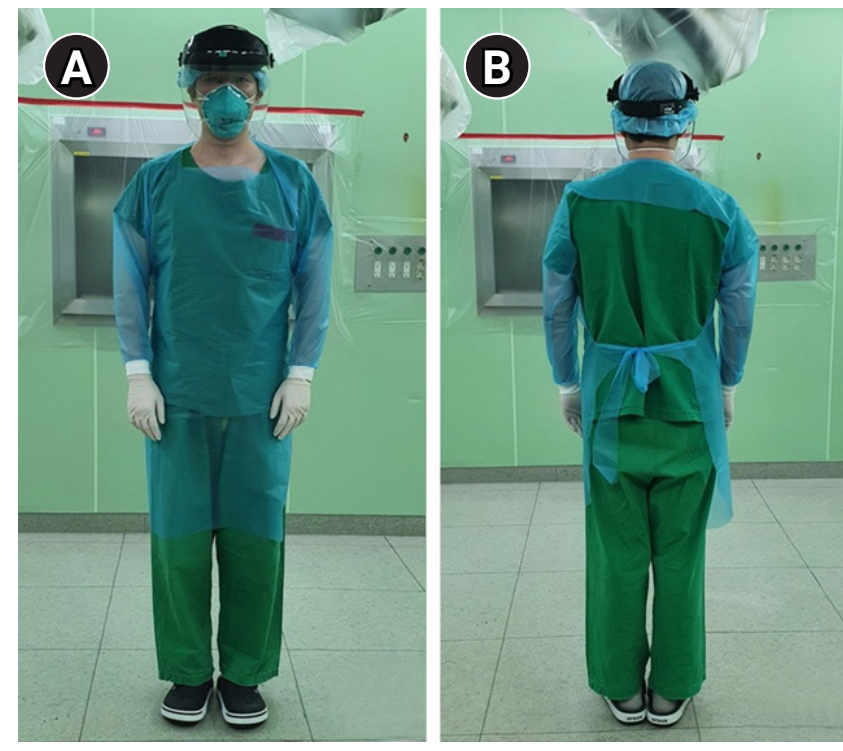

Fig. 5. Standard personal protective equipment in frontal view (A) and back view (B).
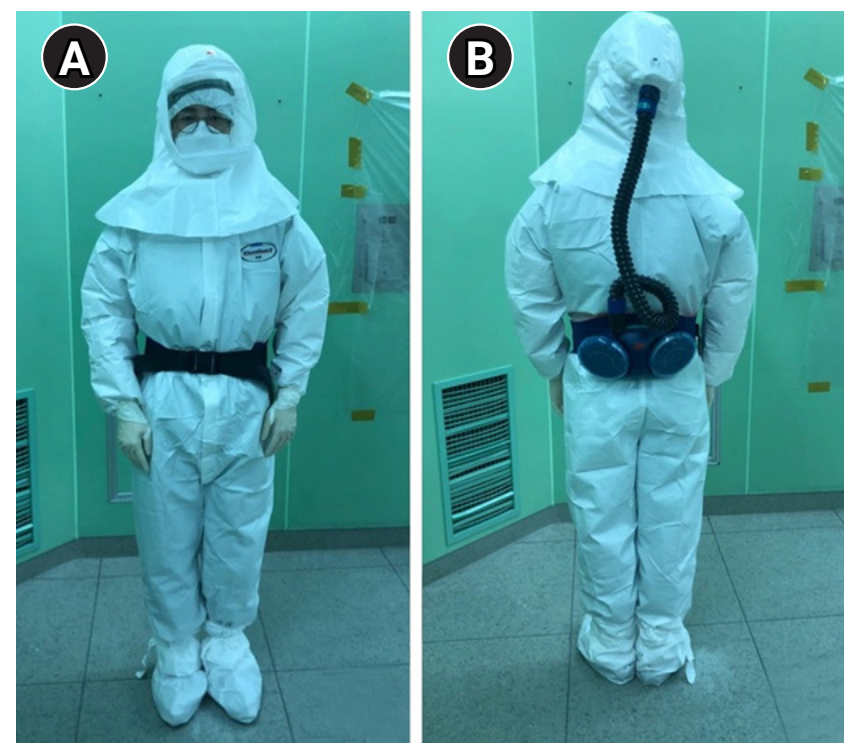

Fig. 6. Enhanced personal protective equipment in frontal view (A) and back view (B).

thesia was successfully performed without healthcare workers being exposed to the risk of infection.

In case 2, healthcare workers did not wear the PPEs properly, resulting in a situation in which they were exposed to risks of infection to COVID-19-related patients. We prevented further exposure to infection by immediate isolation and continuous monitoring of the exposed healthcare workers. Furthermore, staff training sessions about wearing PPEs for healthcare workers were intensified and increased.

After excisional biopsy, the patient was transferred to a different hospital room Another COVID-19 confirmed patient was using a nebulizer in the room. It is thought that this nebulizer use caused airborne viral spread and transmission to other patients in that hospital room. SARSCoV-2 RT-PCR tests were performed on patients, their caregivers, and all staff in this ward as COVID-19 confirmed cases occurred. Those who showed positive results of SARS-CoV-2 RT-PCR were moved to isolation rooms and those who came into contact with COVID-19 patients were isolated immediately for 14 days.

Since then, out hospital has restricted the transfer of patients between hospital rooms to prevent in-hospital transmission.

With COVID-19 infected patients, fever is the first symptom mainly observed, and mild symptoms such as cough and fatigue may occur as well as severe complications such as pneumonia, acute respiratory distress syndrome, cardiac injury, and multi-organ failure $[12,13]$. If only fever occurs, it might be difficult to differentiate the cause of the fever from the other diseases of the patient. In addition, symptoms may occur 5 to 6 days after infection and up to 14 days after transmission. Transmission has been reported even in this subclinical period $[4,14]$. With patients in this subclinical period, it is difficult to clearly determine COVID-19 infection with symptoms or chest images only. Therefore, in areas where COVID-19 is an epidemic, efforts to identify COVID-19 should be made.

In case 2, the patient also had a fever. However, there were no symptoms other than those leading to the surgery. Nothing had changed from the chest radiography 3 days prior to the surgery, and negative results were confirmed on the previously tested SARS-CoV-2 RT-PCR. Therefore, after consulting with the division of infectious disease and infection control department, we excluded the possibility of COVID-19 and performed surgery. However, since the patient was confirmed to have COVID-19 later, the fever at that time might have been caused by SARS-CoV-2 infection or underlying pathology during the subclinical period. It would be better if the surgery was performed with additional SARS-CoV-2 RT-PCR to ensure the patient was excluded from the possibility of having COVID-19. Since then, our hospital has been testing SARS-CoV-2 RT-PCR within 24 hours before surgery for all patients undergoing surgery.

As COVID-19 had become a pandemic throughout the world, it is thought that the surgery of COVID-19-related patients will also increase. Due to the limited space in the 
operating room, there is as risk of airborne viral spread and higher chance of transmission through contact, which increases the risk of healthcare workers being exposed and of contaminating the operating room.

Therefore, in areas where COVID-19 occurs as epidemics, SARS-CoV-2 RT-PCR should be performed before surgery in patients with symptoms of respiratory infections such as fever, cough, and sputum, or those who cannot completely exclude the possibility of COVID-19. In addition, SARS-CoV-2 RT-PCR testing may be necessary to rule out subclinical infection of SARS-CoV-2 to prevent the possibility of transmission in the hospital.

In addition, efforts are needed to minimize the exposure of healthcare workers. Healthcare workers should wear appropriate PPEs. To minimize aerosol generation during intubation, the tidal volume should be kept as small as possible during manual ventilation using a face mask and videolaryngoscopy should be used. Rapid sequence intubation or intubation after sufficient muscle relaxation should be considered to minimize coughing by the laryngeal reflex.

In addition, to limit transmissions, efforts should be made to prepare trainings about wearing PPE, ways to set up a negative pressure room to prevent contamination of the operating room, and a protocol to prevent in-hospital transmission.

\section{SUPPLEMENTARY MATERIALS}

Supplementary video is available at https://doi.org/ 10.17085/apm.20044.

\section{CONFLICTS OF INTEREST}

No potential conflict of interest relevant to this article was reported.

\section{AUTHOR CONTRIBUTIONS}

Supervision: Sang-Gon Lee, Kwang-Seok Shim. Visualization: Jeongmin Oh. Writing-original draft: Hyunkyum Kim. Writing-review \& editing: Kwang-Seok Shim.

\section{ORCID}

Hyunkyum Kim, https://orcid.org/0000-0002-4539-8257

Jeongmin Oh, https://orcid.org/0000-0001-5685-6287

Sang-Gon Lee, https://orcid.org/0000-0002-1017-0068
Kwang-Seok Shim, https://orcid.org/0000-0001-7847-7042

\section{REFERENCES}

1. COVID-19 [serial on the Internet]. Cheongju: Korea Centers for Disease Control \& Prevention [cited 2020 May 12]. Available from http://ncov.mohw.go.kr/.

2. Huang C, Wang Y, Li X, Ren L, Zhao J, Hu Y, et al. Clinical features of patients infected with 2019 novel coronavirus in Wuhan, China. Lancet 2020; 395: 497-506.

3. de Wit E, van Doremalen N, Falzarano D, Munster VJ. SARS and MERS: recent insights into emerging coronaviruses. Nat Rev Microbiol 2016; 14: 523-34.

4. Chan JF, Yuan S, Kok KH, To KK, Chu H, Yang J, et al. A familial cluster of pneumonia associated with the 2019 novel coronavirus indicating person-to-person transmission: a study of a family cluster. Lancet 2020; 395: 514-23.

5. World Health Organization. Infection prevention and control during health care when COVID-19 is suspected [serial on the Internet]. 2020 Mar 19 [cited 2020 May 12]. Available from https://www.who.int/publications/i/item/infection-prevention-and-control-during-health-care-when-novel-coronavirus-(ncov)-infection-is-suspected-20200125.

6. Tran K, Cimon K, Severn M, Pessoa-Silva CL, Conly J. Aerosol generating procedures and risk of transmission of acute respiratory infections to healthcare workers: a systematic review. PLoS One 2012; 7: e35797.

7. Park J, Yoo SY, Ko JH, Lee SM, Chung YJ, Lee JH, et al. Infection prevention measures for surgical procedures during a Middle East respiratory syndrome outbreak in a tertiary care hospital in South Korea. Sci Rep 2020; 10: 325.

8. Wong HYF, Lam HYS, Fong AH, Leung ST, Chin TW, Lo CSY, et al. Frequency and distribution of chest radiographic findings in COVID-19 positive patients. Radiology 2020. doi: 10.1148/ radiol.2020201160. [Epub ahead of print].

9. Xie X, Zhong Z, Zhao W, Zheng C, Wang F, Liu J. Chest CT for typical 2019-nCoV pneumonia: relationship to negative RTPCR testing. Radiology 2020. doi: 10.1148/radiol.2020200343. [Epub ahead of print].

10. Hong KH, Lee SW, Kim TS, Huh HJ, Lee J, Kim SY, et al. Guidelines for laboratory diagnosis of coronavirus disease 2019 (COVID-19) in Korea. Ann Lab Med 2020; 40: 351-60.

11. van Doremalen N, Bushmaker T, Morris DH, Holbrook MG, Gamble A, Williamson BN, et al. Aerosol and surface stability of SARS-CoV-2 as compared with SARS-CoV-1. N Engl J Med 2020; 382: 1564-7.

12. Greenland JR, Michelow MD, Wang L, London MJ. COVID-19 
infection: implications for perioperative and critical care physicians. Anesthesiology 2020; 132: 1346-61.

13. Guan W, Ni Z, Hu Y, Liang W, Ou C, He J, et al. Clinical characteristics of coronavirus disease 2019 in China. N Engl J Med
2020; 382: 1708-20.

14. Li Q, Guan X, Wu P, Wang X, Zhou L, Tong Y, et al. Early transmission dynamics in Wuhan, China, of novel coronavirus-infected pneumonia. N Engl J Med 2020; 382: 1199-207. 
